\title{
GROUND-STATE PROPERTIES AND SPIN WAVES IN ULTRATHIN Fe FILMS COVERED WITH MAGNETIC AND NONMAGNETIC MATERIALS
}

\author{
R. ŚWIRKowICZ \\ Institute of Physics, Warsaw University of Technology \\ Koszykowa 75, 00-662 Warszawa, Poland
}

(Received March 7, 1995)

\begin{abstract}
Properties of ultrathin films consisted of 5 and 7 atomic layers of $\mathrm{Fe}$ and covered with magnetic $(\mathrm{Co})$ or nonmagnetic $(\mathrm{Cu}, \mathrm{Ag})$ materials are investigated within the framework of the multiband model. Ground-state results consistent with those known from $a b$ initio approaches are obtained. Spin waves are studied in the random phase approximation with the use of the susceptibility method. Amplitudes and dispersion relations are calculated. Two acoustic modes with amplitudes enhanced at the interface are found.

PACS numbers: 75.10.Lp, 75.30.Ds, 75.70.-i
\end{abstract}

\section{Introduction}

Magnetic properties of ultrathin Fe films have been intensively studied during the last few years with the use of the Mössbauer spectroscopy [1, 2]. Profiles of the hyperfine field and the temperature dependence of the local field in the spin-wave regime have been determined.

The theory based on the itinerant electron model seems to be the most required approach to the problem of spin waves in transition metal thin films [3, 4]. As far as Fe films are concerned the problem has been undertaken for an unsupported monolayer [5] and for the ultrathin film with free surfaces [6]. The spin-wave dispersion relation was found and the stiffness parameter $D$ was calculated $[5,6]$.

Experiments are mainly performed for films with coated surfaces. The aim of the present paper is to study an influence of the overlayers on the ground-state properties and spin waves in ultrathin Fe films. Systems composed of a few Fe layers and covered with transition magnetic (Co) or nonmagnetic $(\mathrm{Cu}, \mathrm{Ag})$ metals are investigated. 


\section{Method of calculations}

A general Hamiltonian for the system under consideration is taken in the form

$$
\begin{aligned}
H= & \sum_{\nu j m \sigma} E_{\nu} c_{\nu j m \sigma}^{+} c_{\nu j m \sigma}+\sum_{\substack{\nu \nu_{\nu}^{\prime} j^{\prime} \\
m m^{\prime} \sigma}} H_{m m^{\prime}}^{\nu \nu^{\prime}}\left(j, j^{\prime}\right) c_{\nu j m \sigma}^{+} c_{\nu^{\prime} j^{\prime} m^{\prime} \sigma} \\
& +\frac{1}{2} \sum_{\substack{\nu j_{m \sigma \sigma^{\prime}} \\
m m^{\prime} m_{1} m_{1}^{\prime}}} U_{m m_{1}^{\prime} m_{1} m^{\prime}}^{\nu} c_{\nu j_{m \sigma}}^{+} c_{\nu j m_{1}^{\prime} \sigma^{\prime}}^{+} c_{\nu j m_{1} \sigma^{\prime}} c_{\nu j m^{\prime} \sigma}
\end{aligned}
$$

where $c_{\nu j_{m \sigma}}$ denotes an annihilation operator of an electron with spin $\sigma$ and orbital index $m$ at the lattice point $\nu j . E_{\nu}$ is the one-electron potential in the layer $\nu$ and $H_{m m^{\prime}}^{\nu \nu^{\prime}}\left(j, j^{\prime}\right)$ are two-center hopping integrals. The Slater-Koster parametrization with values of parameters which correspond to canonical bands is used. Elements of matrix $U$ describe the effective intra-atomic interactions between electrons in the layer $\nu$. It is assumed that integrals which represent the effective Coulomb and exchange interactions in the same and different orbital states are of the same value $[5,6]$.

Similarly as in Refs. $[5,6]$ calculations are performed in the tight-binding approximation with five orbitals of symmetry $d$ taken into account. It is known that in the case of bulk Fe $s p-d$ hybridization plays an important role and strongly influences the band structure and spin-wave energies [7]. Negative values of the parameter $D$ are obtained when the effects of hybridization are neglected. However, in the case of ultrathin films the presence of the surface leads to essential modifications of the band structure and to dehybridization of $s p$ and $d$ states [8]. Calculations performed in Refs. [5, 6] clearly show that it is possible to get positive $D$ when a film is thin enough. The influence of the hybridization becomes stronger with an increase in a number of atomic layers. The value of $D$ calculated in Ref. [5] is about $20 \%$ lower than that obtained for a bulk sample. Similar lowering of $D$ was found for $\mathrm{Ni}$ monolayer, though hybridization effects can be completely neglected in the case of nickel [5].

The band structure of the film is calculated self-consistently in the HartreeFock (IIF) approximation. Then, the dynamic susceptibility method is used to investigate spin waves, which propagate in the system. The transverse susceptibility of a thin film is calculated within the framework of the random phase approximation (RPA) and the mixed Bloch-Wannier representation is used [9]. Evaluations are performed for various values of a two-dimensional wave vector $\boldsymbol{q}$ parallel to the surface.

\section{One-electron ground-state properties}

Numerical calculations are performed for systems which consist of 5 or 7 atomic layers of $\mathrm{Fe}$ and are covered with a layer of $\mathrm{Cu}, \mathrm{Ag}$ or $\mathrm{Co}$ metal. The bcc structure with the same lattice constant is assumed for the whole system. The surface of the film is assumed to be perpendicular to the [001] direction. Slater-Koster 
parameters appropriate for a given material are taken. The parameters which correspond to hopping between two different materials are taken in the form of the geometrical mean. The integral $U$ equal to $0.88 \mathrm{eV}$ is chosen for Fe planes [7] and it is slightly modified in the surface layers. Values of the potential $E_{\nu}$ are chosen in such a way that the correct number of $d$-electrons is obtained. The calculated total number of electrons in Fe layers is close to 7.00 and charge transfers are small (less than $0.1 \mathrm{eV}$ ). The number of electrons in the surface layer equal to 8.04, 9.94, 9.97 is obtained for $\mathrm{Co}, \mathrm{Cu}$ and $\mathrm{Ag}$, respectively.

Then, the local density of states and profiles of the magnetization are calculated. Curves obtained for the surface, subsurface and central layers in the case of $\mathrm{Ag}, \mathrm{Cu}$ and Co overlayers are presented in Figs. 1a, b and c, respectively. Surface states corresponding to $\mathrm{Ag}$ lay far below the Fermi level and they do not influence $\mathrm{Fe}$ states considerably. Because of the weak hybridization in the central part of the DOS calculated for the subsurface Fe layer high peaks can be seen which correspond to localized electron states. For the minority of spins the peak appears at the Fermi level. To some extent the curves resemble results obtained for the free Fe surface. On the other hand, the density of states (DOS) of the central layer and especially the curve for the minority of spins with the small peak at the Fermi level is similar to the one characteristic for the bulk Fe. Results obtained for the film covered with $\mathrm{Cu}$ are similar to those given in Fig. 1a. The presence of the electron states localized at the $\mathrm{Fe}-\mathrm{Ag}(\mathrm{Cu})$ interface leads to a considerable enhancement of the magnetization in the subsurface layer. The magnetic moment calculated for this layer is equal to 2.67 and 2.76 for $\mathrm{Ag}$ and $\mathrm{Cu}$ overlayers, respectively. These values of the subsurface magnetization are slightly lower than the one characteristic for the free Fe surface. In the inner part of the film the magnetic moment decreases taking value close to 2.2 in the central layer. A small magnetic moment is induced in the nonmagnetic $\mathrm{Cu}$ and $\mathrm{Ag}$ overlayers. The presented results are well consistent with those obtained within the framework of the $a b$ initio approach [10].

The interaction between Co and Fe $d$-bands is much stronger and it influences the density of states (Fig. 1c) and the magnetization in the Fe subsurface layer. The magnetic moment at the interface is equal to 2.41 and it is smaller than in the case of $\mathrm{Cu}, \mathrm{Ag}$ metals. For the Co overlayer the value of 1.62 is found, which is close to the magnetization of the bulk material.

It should be also pointed out that the number of holes with the majority of spins is reduced in the Fe subsurface layer as compared to the central one. The effect can be observed for Co and nonmagnetic overlayers. It is especially distinctive in the case of $\mathrm{Cu}$. The reduction of the holes with the majority of spins near the surface or the interface is the result known from the more sophisticated approaches $[8,10]$.

\section{Dynamic susceptibility and spin waves}

The transverse dynamic susceptibility is calculated for various values of the wa ve vector $q$ parallel to the surface. $\operatorname{Im} \chi$ obtained for the subsequent atomic layers is presented in Fig. 2 for the film coated with $\mathrm{Cu}$. Only curves found for $\mathrm{Fe}$ layers are given. The peaks which appear in $\operatorname{Im} \chi$ correspond to the low-lying spin-wave 

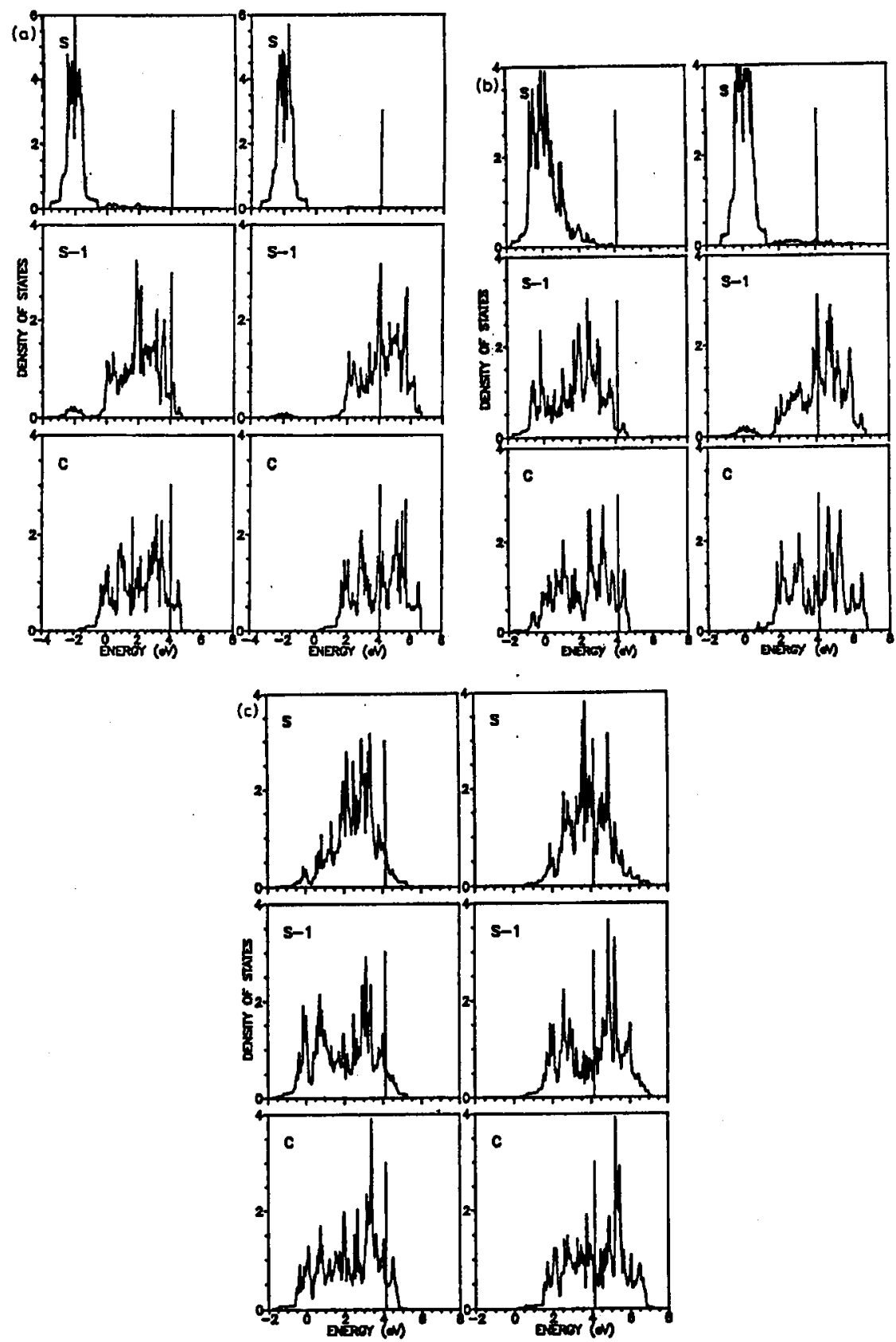

Fig. 1. Density of states for the surface, subsurface and central layers in the case of the film covered with $\mathrm{Ag} \mathrm{(a),} \mathrm{Cu}$ (b), Co (c). The vertical straight lines correspond to the Fermi energy. 


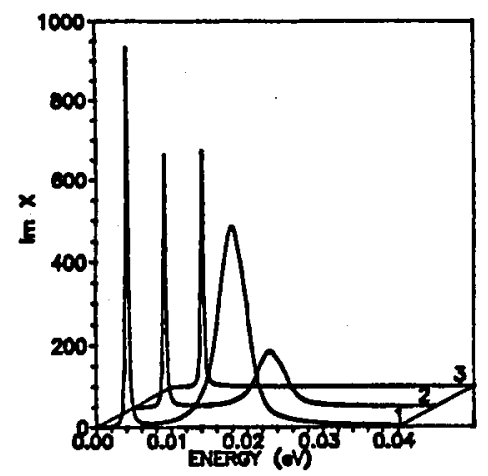

Fig. 2. $\operatorname{Im} \chi$ calculated for the subsequent Fe layers in the film covered with $\mathrm{Cu}$.

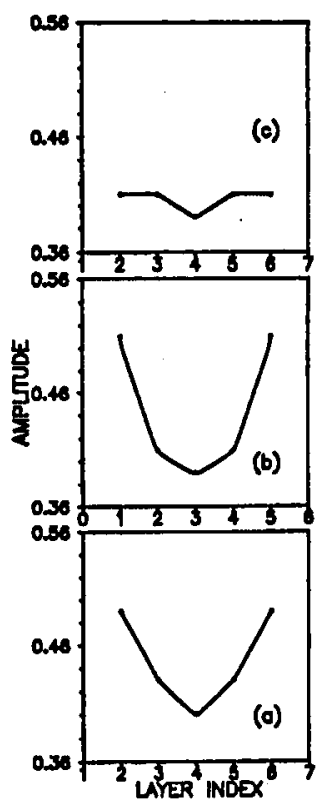

Fig. 3. Amplitudes of the spin-wave mode with the lowest energy for films covered with $\mathrm{Cu}$ (a), with free surfaces (b), covered with Co (c).

modes. Very high peaks can be seen for the subsurface Fe layer. The peaks height decrease in inner Fe planes, showing a decrease in the spin-wave amplitudes. The profile of the amplitude of the mode with the lowest energy calculated for the subsequent $\mathrm{Fe}$ planes in the film with $\mathrm{Cu}$ overlayers is presented in Fig. 3a. For comparison the profile obtained for the Fe film with free surfaces is also given in the figure (Fig. 3b). One can see that in both cases the amplitude is strongly enhanced in the outer Fe layer showing a tendency of the mode to localization near the surface or interface. Similar enhancement of the amplitude is obtained for the second mode. So, two acoustic modes with high amplitudes at the surface 
or interface layers are found. The enhancement of the amplitude of the two lowest modes is also obtained for the $\mathrm{Fe} / \mathrm{Ag}$ interface. However, in the case of the film covered with Co overlayer (Fig. 3c) the amplitude of the first mode is only slightly changed at the interface. It can be a result of the strong hybridization between $\mathrm{Fe}$ and Co $d$-bands.

Dispersion relations calculated for systems under consideration are presented in Figs. 4a, b, c. Two branches which correspond to the modes with the lowest energies are well seen in all cases. Energies of the third mode, the volume one, are higher, especially for the Ag overlayer.
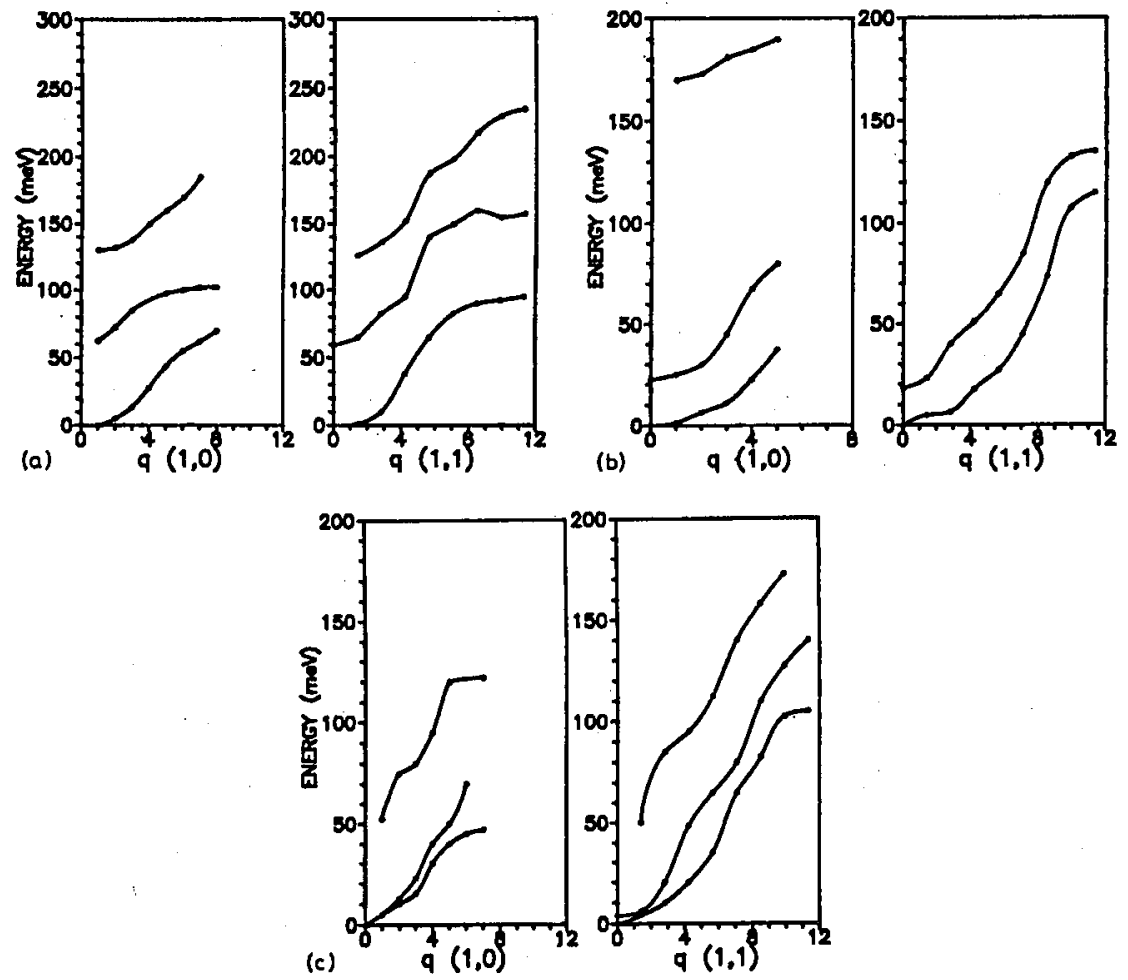

Fig. 4. Spin-wave dispersion relations for [ $\left[\begin{array}{ll}1 & 0\end{array}\right]$ and $\left[\begin{array}{ll}1 & 1\end{array}\right]$ directions. Film covered with $\mathrm{Cu}$ (a), Ag (b), Co (c).

In the region of small values $q$ the quadratic relation is well fulfilled at least for two low-lying modes. The plot of the spin-wave energy in dependence on $q^{2}$ for the film covered with Co is presented in Fig. 5. Calculated points fit the straight line very well. The stiffness parameter $D$ determined by the gradient of the line is equal to $94 \mathrm{meV} \AA^{2}$ for the mode which propagates in the [1 0 ] direction. The value of $D$ obtained for the second mode is higher and equal to $127 \mathrm{meV} \AA^{2}$. Calculations performed for the film with free surfaces and consisting of $5 \mathrm{Fe}$ layers give the spin-wave parameter of the value $72 \mathrm{meV} \AA^{2}$ for the first mode [6]. The 


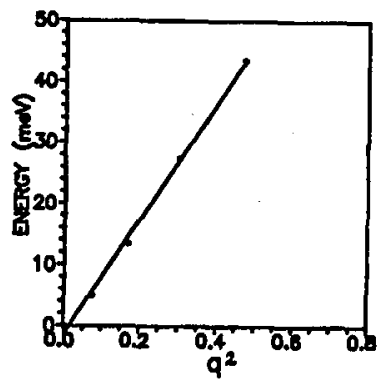

Fig. 5. Spin-wave energy in dependence on $q^{2}$ for the film covered with Co.

fact that higher $D$ is obtained for films covered with Co is in agreement with results known for $\mathrm{Fe}-\mathrm{Co}$ alloys [7]. The value of the stiffness parameter calculated for the lowest mode, which can propagate in the [1 0$]$ direction in the film covered with $\mathrm{Cu}$ overlayer, is equal to $79 \mathrm{meV} \AA^{2}$. It is very close to the one obtained for the Fe film with free surfaces. In both cases the spin-wave mode shows a similar tendency to localization (Fig. 3). The parameter $D$ found for the film covered with the $\mathrm{Ag}$ overlayer is relatively small and equal to $58 \mathrm{meV} \AA^{2}$ for the first mode and the $\left[\begin{array}{ll}1 & 0\end{array}\right]$ direction. However, in this case the thicker film consisting of $7 \mathrm{Fe}$ layers has been taken into account.

\section{Concluding remarks}

The approach presented in the paper enables one to describe in the proper way ground-state properties of ultrathin Fe films covered with overlayers. The obtained results are well consistent with those known from $a b$ initio theories. The magnetization at the $\mathrm{Fe}-\mathrm{Cu}(\mathrm{Ag})$ boundary is considerably enhanced what is the result of the presence of localized electron states.

As far as spin waves are concerned two acoustic modes relatively well localized at the interface are found when mixing of $d$-bands of the overlayer and the $\mathrm{Fe}$ substrate is very weak ( $\mathrm{Cu}, \mathrm{Ag}$ overlayers). In the case of the strong interaction the enhancement of the interface amplitude is not observed (Co overlayer).

Calculations of the dispersion relations show that in a region of small wave vectors the quadratic relation is quite well fulfilled for low-lying modes. The results are qualitatively consistent with those obtained within the framework of the Heisenberg model. The calculated dispersion relations are relatively flat. The presence of the localized acoustic modes can lead to the considerable lowering of spin-wave energies [11], however, the values of the parameter $D$ seem to be too small as compared to the bulk one. It can be considered as a result of the fact that $s p-d$ hybridization has been neglected in the approach $[5,6]$. The smallest value of $D$ is obtained in the paper for the film consisting of 7 atomic planes of $\mathrm{Fe}$ and covered with Ag. Calculations performed for Fe film with free surfaces and consisting of 7 layers give negative value of $D$ for the first mode [6].

The fact that essentially different results are obtained for films consisting of $7 \mathrm{Fe}$ layers but with free surfaces or covered with $\mathrm{Ag}$ overlayers shows that the 
parameter $D$ is strongly dependent on the band structure and small modifications can be important.

The result obtained in the paper is consistent with the conclusion drown in Ref. [6] that the influence of hybridization effects on the spin-wave energies becomes stronger with an increase in a number of atomic layers in the film. It can be understood that in thicker films volume effects appear to be more important than the surface ones.

\section{References}

[1] M. Przybylski, J. Korecki, U. Gradmann, Appl. Phys. A 52, 33 (1991).

[2] G. Bayreuther, Hyperfine Interact. 47, 237 (1989).

[3] J. Mathon, S.B. Ahmad, Phys. Rev. B 37, 660 (1988).

[4] R. Świrkowicz, Physica B 167, 239 (1990).

[5] J. d'Albuquerque, D.M. Edwards, J. Mathon, R.B. Muniz, J. Magn. Magn. Mater. 93, 295 (1991).

[6] R. Świrkowicz, A. Sukiennicki, Vacuum 46, 551 (1995).

[7] R.B. Muniz, J.F. Cooke, D.M. Edwards, J. Phys. F 15, 2357 (1985).

[8] S. Ohnishi, A.J. Freeman, Phys. Rev. B 28, 6441 (1983).

[9] R. Świrkowicz, Phys. Status Solidi B 129, 641 (1985).

[10] A.J. Freeman, C.L. Fu, J. Appl. Phys. 61, 3356 (1987).

[11] H. Puszkarski, in: Progress in Surface Science, Ed. S.G. Davison, Vol. 9, Pergamon, New York 1979, p. 191. 